\title{
Onboard Flow Sensing for Downwash Detection and Avoidance with a Small Quadrotor Helicopter
}

\author{
Derrick W. Yeo, ${ }^{*}$ Nitin Sydney ${ }^{\dagger}$ and Derek A. Paley ${ }^{\ddagger}$ \\ University of Maryland, College Park, Maryland, MD 48109, U.S.A \\ Donald Sofge ${ }^{\S}$ \\ Naval Research Laboratory, Washington, DC 20375 , U.S.A
}

\begin{abstract}
Small rotary-wing UAVs are susceptible to gusts and other environmental disturbances that affect inflow at their rotors. Inflow variations cause unexpected aerodynamic forces through changes in thrust conditions and unmodeled blade-flapping dynamics. This paper introduces an onboard, pressure-based flow measurement system developed for a small quadrotor helicopter. The probe-based instrumentation package provides spatially distributed airspeed measurements along each of the aircraft-fixed axes. Lateral and vertical windspeed estimates enable the development of disturbance-tolerant flight control strategies. The focus of this paper is vertical flow disturbances such as those caused by the downwash of a second vehicle. Real-time velocity measurements are incorporated into a recursive Bayesian estimator to localize a nearby rotorcraft using its downwash. A path planner developed for proximity flight is demonstrated through indoor flight testing with multiple vehicles to safely guide an instrumented quadrotor towards a goal point while avoiding another quadrotor.
\end{abstract}

\section{Nomenclature}

$u, v, w$ Velocity components, $\mathrm{m} / \mathrm{s}$

$L_{u, v, w, b}$ Velocity calibration factor

$\mathrm{P}_{i} \quad$ Port pressure, in $\mathrm{H}_{2} \mathrm{O}$

$\rho \quad$ Air density, $\mathrm{kg} / \mathrm{m}^{3}$

$\beta \quad$ External source location vector

$\boldsymbol{z}\left(t_{k}\right) \quad$ Flow speed measurement vector

$A \quad$ Posterior scaling constant

$\mu \quad$ Dynamic viscosity, $\mathrm{kg} /(\mathrm{ms})$

$W_{0} \quad$ Velocity constant

$r \quad$ Radial distance from center line

$u_{x, y} \quad$ Control input

$K \quad$ Control gain

$J \quad$ Cost function

$k_{J} \quad$ Optimization weighting variable

$x, y \quad$ Quadrotor position

Subscript

$i \quad$ Probe number

$k \quad$ Time step

$s \quad$ Disturbance source index

\footnotetext{
*Research Associate, Aerospace Engineering Department, dyeo@umd.edu , AIAA Member.

${ }^{\dagger}$ Ph.D Candidate, Aerospace Engineering Department, nsydney@umd.edu, AIAA Student Member.

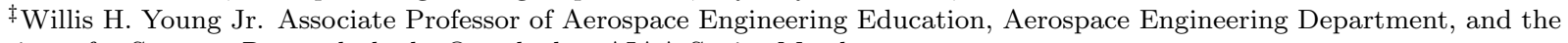
Institute for Systems Research dpaley@umd.edu, AIAA Senior Member.

$\S$ Computer Scientist, Naval Research Laboratory, donald.sofge@nrl.navy.mil , AIAA Member.
} 


\section{Introduction}

By using four independent rotors to provide lift and control moments, the quadrotor helicopter provides researchers with a small, mechanically simple and effective vertical-flight platform. Typically, the lift and resultant torque due to each rotor can be approximated with simple aerodynamic models. Although these models have proven to be adequate in low advance-ratio flight conditions, ${ }^{1}$ they cannot account for the flow conditions associated with high-speed flight or the presence of large disturbances such as wind gusts or downwash from nearby rotorcraft. Real-time measurements of the flow field around a flying vehicle provide a description of the flight conditions that would otherwise be impossible with only inertial-based instruments.

This paper introduces a pressure-probe flow measurement system developed for a small quadrotor. The design, fabrication and calibration of the instrumentation package are presented along with data from autonomous flight trials using motion-capture based positioning data. As a demonstration of the capabilities afforded by onboard flow sensing, a strategy for avoiding the downwash of a neighboring rotorcraft is proposed and demonstrated.

Assimilating vertical flow field measurements with a Bayesian estimation algorithm detects and localizes the source of vertical flow disturbances for use in a flight-path planner developed for proximity flight. The path planner minimizes a cost function that drives the vehicle to a desired waypoint while avoiding the vertical downwash. Test results from indoor experiments with two quadrotors operating at different altitudes showcase the capability of the flight planner to generate trajectories that successfully avoid the downwash of a second rotorcraft.

The contributions of the research described in this paper are (1) implementation of an onboard flow sensing instrumentation system that uses an array of distributed pressure sensors to produce measurements of the surrounding flow field; and (2) a flight-path planner that estimates the surrounding flow field in order to generate a safe trajectory to the desired goal location. The measurements from the instrumentation system are used in a recursive Bayesian estimator to estimate the position of another quadrotor, which in turn is used in the path planner for guidance and control.

The rest of the paper is as follows. A background of onboard flow sensing for small-scale flyers is provided in Section II. Section III describes the design and testing of the air-speed instrumentation system. Section IV presents a recursive Bayesian estimator that estimates the position of a hovering quadrotor using downwash measurements. Section V contains results from experimenting with a flight-path planner for proximity flight. Section VI summarizes the paper and ongoing work.

\section{Background}

The use of flow sensing has been employed with great success on naturally evolved flyers. ${ }^{2}$ Using distributed measurements of relative wind, sufficiently large creatures such as birds align themselves with their intended direction of travel and account for the effects of wind gusts. By detecting fine details of the ambient flow field, small creatures like insects improve their flight performance by finely tuning their flap stroke to suit flight conditions. ${ }^{2}$ Gewecke and Woike ${ }^{3}$ showed that directing airflow over avian feathers could cause steering impulses, and as shown in more recent work by Brown and Fedde, ${ }^{4}$ birds have the necessary sensor-feather mechanisms in the wing to predict stall and measure airspeed.

In contrast to natural flyers, the current paradigm of small UAS instrumentation is to integrate inertial measurements supplemented by (scalar) airspeed. A five-hole probe providing air-data measurements that include airspeed, angle of attack, and sideslip has been successful in applications involving conventional fixed wing flight within the traditional flight envelope. ${ }^{5-7}$ These platforms provide a baseline capability for more advanced tests in areas such as cooperative control $^{8}$ and ocean-borne operations ${ }^{9}$ for both fixed-wing and rotary-wing vehicles. ${ }^{10,11}$

Seeking improved platform performance, researchers have looked to expand the notion of onboard flow sensing and apply it to various levels of vehicle control. For example, flow information can be used to finetune aerodynamic parameters for performance. Patel and Corke $^{12,13}$ considered the time-domain response from a high-bandwidth pressure sensor to predict incipient flow separation at the wing leading edge and trigger a plasma flow actuator to alleviate flow separation.

Flow sensing can also improve flight control. Xu et al. ${ }^{14}$ implemented arrays of micro-machined shearstress sensors on the leading edge of a low aspect-ratio delta wing. The sensor system was developed to support control strategies that effected aerodynamic flight control through boundary-layer manipulation. ${ }^{15}$ 
The AVOCET project ${ }^{16}$ aims to continuously tailor the pressure distribution and resulting forces and moments across the wing using advanced micro-tuft sensors and hybrid fluidic flow actuators. Under attached flow conditions, NASA has supported wind tunnel-based implementation and testing of a distributed actuation and sensing array for use on a blended-wing-body UAV, using a series of pressure measurements to study the effectiveness of a morphing-wing control strategy. ${ }^{17,18}$ More recently, Watkins et al. have demonstrated improved attitude control of a small fixed-wing vehicle in turbulent flow conditions through the integration of pressure sensors embedded in the main wings.

At a higher level, aerodynamic sensing provides information that can drive vehicle configuration or guidance decisions based on flight conditions. For example, Cox et al. ${ }^{19}$ used pressure based estimates of the lift curve above an airfoil as feedback for an automated cruise flap. Yeo et al. ${ }^{20}$ used real time stall detection through pressure sensing to change controller modes on a small, fixed-wing UAV during transition between forward flight and propeller-borne hover.

The work presented in this paper seeks to expand the notion of onboard aerodynamic sensing for small rotorcraft UAVs. Additional flow information across the rotors promises to improve quadrotor attitude stabilization in wind ${ }^{21}$ while also detecting environmental disturbances for improved trajectory planning. This paper focuses on aerodynamic sensing as an enabler for improved multiple vehicle operations, which often require close-proximity flight.

Recently, there have been several approaches proposed for dealing with proximity flight. ${ }^{21-24}$ For example, ${ }^{21}$ a nonlinear controller that uses estimates of the wind speed to compensate for a rotor downwash impinging on the vehicle. However, the controller assumes knowledge of the structure of the downwash. In another example, ${ }^{24}$ a controller is designed to compensate for minor $(1-2 \mathrm{~m} / \mathrm{s})$ changes in airspeed due to quadrotors operating at the same altitude. In both examples, the external wind (or an estimate of the wind) impinging on the rotorcraft is assumed to be available.

External wind information is provided in this paper by a novel probe-based flow measurement package. Unlike traditional measurement techniques employed on small rotors such as hot-wire anemometry ${ }^{12}$ and optical velocimetry methods, ${ }^{13,17,19}$ the pressure-probe-based approach used here is inherently portable, rugged and well-suited for implementation onboard a small flight vehicle. The instrumentation system presented in this work is capable of providing flow speed measurements below $5 \mathrm{~m} / \mathrm{s}$ in real time, enabling the implementation of flow-based guidance and control. To show the utility of the instrumentation system, we design a flight-path planner to avoid downwash of another quadrotor while simultaneously directing the vehicle toward a goal location.

\section{Quadrotor Instrumentation System}

The flow instrumentation system shown in Fig. 1 uses custom-built pressure probes that provide flow information through differential-pressure measurements. A single top-mounted airspeed probe takes measurements along the longitudinal and lateral directions of the vehicle and four vertical-airspeed probes are mounted uniformly around the vehicle outside the downwash the rotors. A two-port, fore- and aft-facing configuration allows for flow speeds under $5 \mathrm{~m} / \mathrm{s}$ to be measured with improved signal-to-noise-ratio as compared to a standard pitot-static probe configuration.

The instrumentation packages are portable and easily mounted on a variety of small quadrotor vehicles. The probes are constructed out of aluminum tubing that is bent to form two to four pressure ports at each tip. The probe design is based on a multi-hole probe for propeller-wash measurements. ${ }^{10}$ The design, geometry and port configuration of the various probes is shown in Fig. 1. The airspeed component across the pressure port pairs are related to the differential pressure measurement by

$$
\begin{gathered}
u=L_{u} \sqrt{\frac{2 L_{b}\left(P_{1}-P_{2}\right)}{\rho}} \\
v=L_{v} \sqrt{\frac{2 L_{b}\left(P_{4}-P_{3}\right)}{\rho}} \\
w_{i}=L_{w_{i}} \sqrt{\frac{2 L_{b}\left(P_{i, 1}-P_{i, 2}\right)}{\rho}},
\end{gathered}
$$




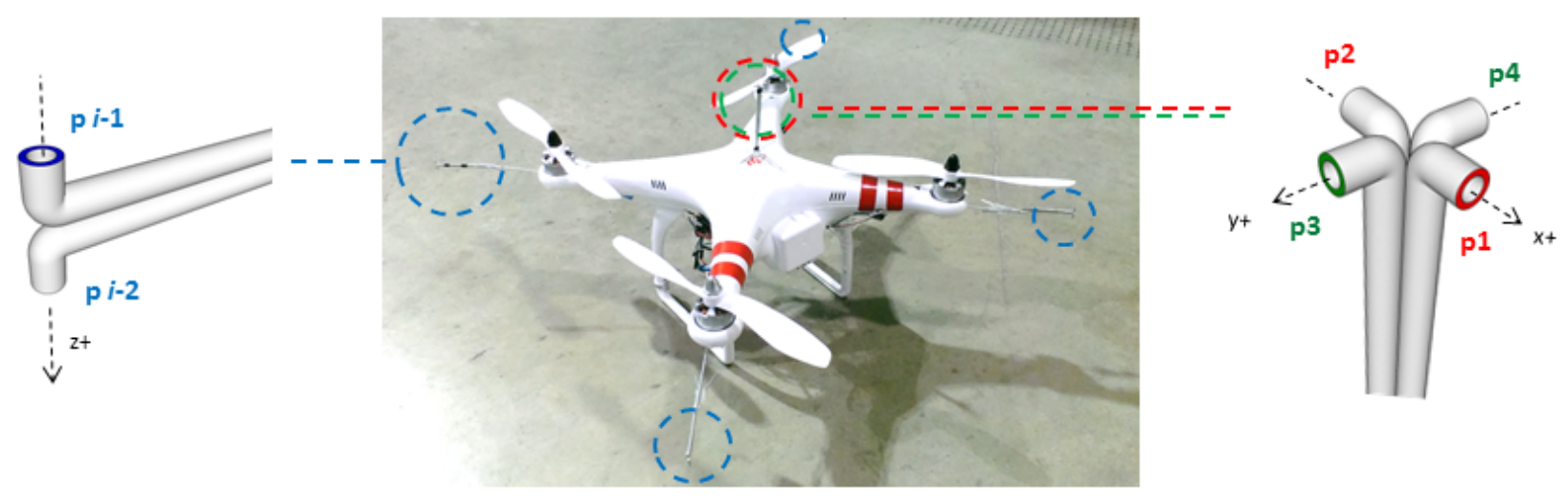

Figure 1. Quadrotor flow instrumentation probe locations and geometry.

where the scaling factors $L_{u}, L_{v}$ and $L_{w}$ are determined by calibration in a wind tunnel and the factor $L_{b}$ includes the effect of gravity and unit conversion. The calibration is applied onboard and a moving average filter is implemented. (The air data system is built around a Freescale Semiconductor MK20DX256VLH7 ARM Cortex M4 microprocessor running 13bit ADC at 1kHz. Honeywell HSCDRRN001NDAA3 differential pressure sensors are used to measure probe pressures and processed flow speed measurements are transmitted over a serial port interface.)

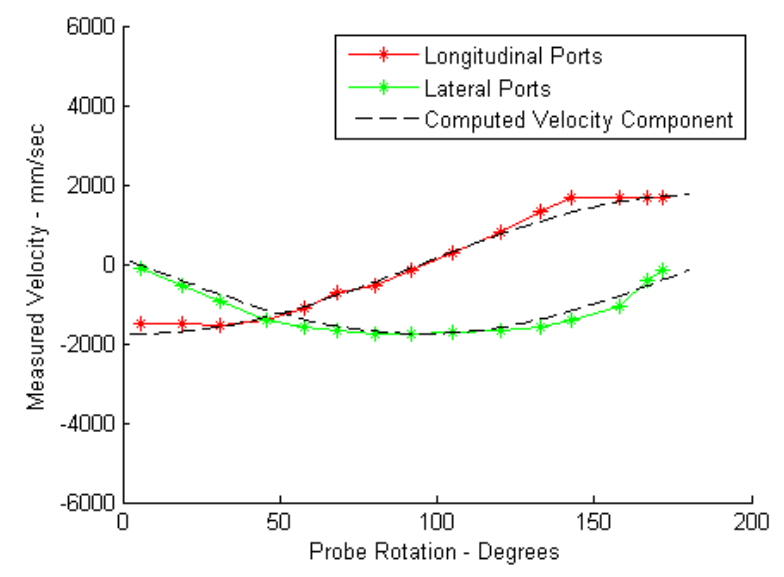

(a) $1.8 \mathrm{~m} / \mathrm{s}$ Freestream

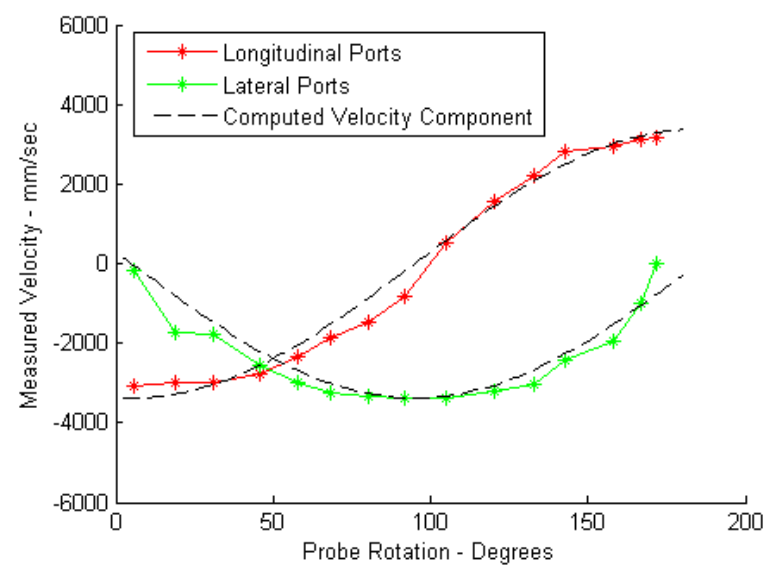

(b) $3.3 \mathrm{~m} / \mathrm{s}$ Freestream

Figure 2. Wind tunnel tests: measured longitudinal and lateral airspeed components

The directional sensitivity of the port configuration was first evaluated through wind-tunnel testing. The probe is mounted on a rotating stage that yaws the probe in a constant free stream. Measurements from both sets of ports were compared against idealized wind-vector values at each probe angle. Fig. 2 shows that the probe is able to estimate flow direction reasonably with maximum error under $15 \%$ of full-scale deflection. The ability of the instrumentation package to provide wind-vector information in flight was also tested using remotely piloted maneuvers in an indoor motion-capture facility. A Blade 350QX was flown diagonally across the capture space to generate airspeed along the body-fixed lateral and longitudinal axes. 
Wind-vector measurements taken by the translational probe were streamed to a groundstation and compared against ground-speed data from the motion-capture system, assuming still air.

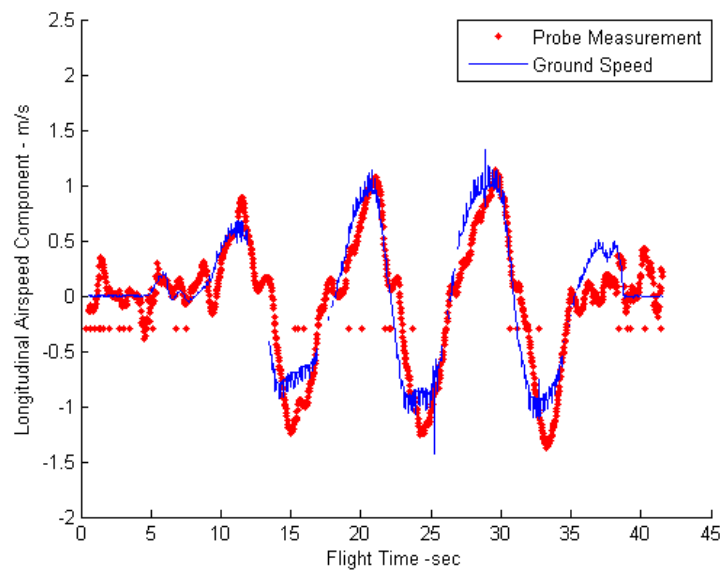

(a) Longitidinal component

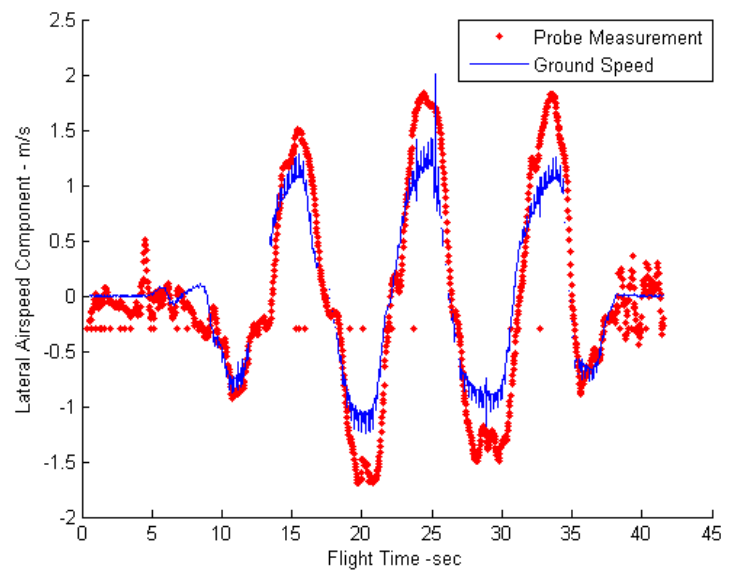

(b) Lateral component

Figure 3. Remotely piloted flight tests: measured longitudinal and lateral airspeed components

Fig. 3 suggests that the pressure-port configuration is capable of providing reliable directional velocity measurements. Using a combination of one lateral airspeed probe and four vertical downwash probes, the instrumentation scheme is capable of providing (relative) wind-vector information over the body of the vehicle. This paper focuses on using the vertical wind-vector measurements to sense vertical flow disturbances.

\section{Localizing the Source of a Rotor Downwash}

Vertical flow disturbances affect any flight vehicle by creating transient disruptions to aerodynamic force generation. For multiple rotorcraft operating in close proximity, impinging downwash from a vehicle operating at a higher altitude causes sudden and possibly catastrophic loss of thrust from the affected rotor. Knowledge of the flow environment provides a flight controller with the data to accommodate and avoid such disturbances. This section summarizes the development of a Bayesian estimation scheme and accompanying vertical flow field model that uses real-time flow measurements around a quadrotor to localize and avoid the downwash from another quadrotor.

Recursive Bayesian estimation provides a probabilistic framework for inferring an unknown quantity given a set of measurements. ${ }^{21}$ An estimator is used here to continuously update the most likely location of an external source of downwash based on a set of vertical flow measurements. The location of the external source is denoted by $\beta=\left(x_{s}, y_{s}\right)$, where $\left(x_{s}, y_{s}\right)$ are the inertial coordinates of a nearby rotorcraft operating at a higher altitude and creating a region of accelerated flow. (We assume the instrumented quadrotor knows its own inertial position too). Let $\boldsymbol{z}\left(t_{k}\right)$ be a vector of noisy windspeed measurements taken using the flow measurement system at time $t_{k}$. The probability of $\beta$ being the center of a vertical flow disturbance given the set of measurements up to $t_{k}$ is

$$
p\left(\beta \mid \boldsymbol{z}\left(t_{1}\right), . ., \boldsymbol{z}\left(t_{k}\right)\right)=A p\left(\boldsymbol{z}\left(t_{k}\right) \mid \beta\right) p\left(\beta \mid \boldsymbol{z}\left(t_{1}\right), . ., \boldsymbol{z}\left(t_{k-1}\right)\right)
$$

where $p\left(\beta \mid \boldsymbol{z}\left(t_{1}\right), . ., \boldsymbol{z}\left(t_{k-1}\right)\right)$ is the posterior probability density and $A$ is a normalization constant that gives the posterior unit integral. The likelihood function $p\left(\boldsymbol{z}\left(t_{k}\right) \mid \beta\right)$ represents the conditional probability of receiving measurements $\boldsymbol{z}\left(t_{k-1}\right)$ given a nearby vehicle located at $\beta$. The mode $\hat{\beta}$ of (4) is the maximum likelihood estimate of the source of downwash. Suppose there are $N$ spatially distributed probes across the vehicle; the $i$ th probe has the likelihood function $p\left(\boldsymbol{z}^{i}\left(t_{k}\right) \mid \beta\right)$, where $\boldsymbol{z}^{i}\left(t_{k}\right)$ is the measurement from the $i$ th probe. The posterior based on all probe measurements is

$$
p\left(\beta \mid \overline{\boldsymbol{z}}\left(t_{k}\right)\right)=p\left(\beta \mid \boldsymbol{z}\left(t_{1}\right), . ., \boldsymbol{z}\left(t_{k-1}\right)\right) \prod_{i=1}^{N} p\left(\boldsymbol{z}^{i}\left(t_{k}\right) \mid \beta\right),
$$


where $\overline{\boldsymbol{z}}\left(t_{k}\right)$ comprises the measurements from all probes up to and including time $t_{k}$.

A key component in implementing a Bayesian estimator is a suitable likelihood function that translates measurements to information of the environment through knowledge of the expected flow field. The likelihood function translates a vertical-velocity measurement to information pertaining to the location of a nearby vehicle through knowledge of the downwash it generates. Obtaining a simple flow model that adequately captures the key characteristics of a range of downwash flow $\mathrm{s}$ is challenging as a rotor-driven flow field is inherently complex, time-varying and characterized by a number of unknown parameters. Also, a simple model for estimation should not require numerical simulation or significant prior knowledge. To this end, a first-order analysis of the governing flow equations is employed here.

Consider a two-dimensional flow field with a rotor generating thrust along the $z$-axis. Velocity components $w$ and $v$ lie along the $z$ and $y$ coordinates respectively. ${ }^{25}$ The momentum equation in the $z$ direction is

$$
\frac{\partial w}{\partial t}+w \frac{\partial w}{\partial z}+v \frac{\partial w}{\partial y}=g_{z}-\frac{1}{\rho} \frac{\partial p}{\partial z}+\frac{\mu}{\rho}\left(\frac{\partial^{2} w}{\partial z^{2}}+\frac{\partial^{2} w}{\partial y^{2}}\right)
$$

where $\rho$ is air density, $\mu$ is the dynamic viscosity of the air, and $g_{z}$ represents body forces due to effects such as bouyancy. The following set of assumptions are applied as follows: (1) the mean flow field is unchanging, so $\partial w / \partial t$ is zero; although rotorwash is highly turbulent, a rotorcraft in a steady flight condition will generate a steady mean flow field velocity. (2) Cross-stream flow is small compared to the downstream velocity; this assumption is based on the intuition that even with the helical structure common to propeller-driven flow $\mathrm{s}$, cross-stream velocity plays a secondary role in thrust generation. For simplicity, only the thrust-aligned velocity component is considered and $\partial w / \partial y$ is neglected. (3) At each $z$ location, the stream-wise variation in $w$ is small compared to the cross stream changes; hence, $\partial^{2} w / \partial z^{2}$ is zero. (4) Lastly, bouyancy and external pressure gradients are not present, so $g_{z}$ and $\partial p / \partial z$ are neglected.

Eq. (6) is further simplified by linearizing about a constant peak velocity $W_{0}$ based on the intuition that center-line flow velocities obey a $1 / z$ decay. The result is

$$
W_{0} \frac{\partial w}{\partial z}=\frac{\mu}{\rho}\left(\frac{\partial^{2} w}{\partial y^{2}}\right)
$$

Solving (7) in cylindrical coordinates yields a Gaussian velocity profile reminiscent of the velocity variation observed in established turbulent jet profiles. ${ }^{26}$ The expected vertical velocity measured at a lateral distance $r(\beta)=\sqrt{\left(x-x_{s}\right)^{2}+\left(y-y_{s}\right)^{2}}$ and a downstream distance $z$ from the center of an idealized rotorcraft is

$$
w(\beta)=\frac{C}{z} W_{0} \exp \left(-\frac{W_{0} r(\beta)^{2} \rho}{4 z \mu}\right) .
$$

The measurement $\boldsymbol{z}^{i}\left(t_{k}\right)$ of the $i$ th sensor at time $t_{k}$ is assimilated into the Gaussian likelihood function

$$
p\left(\boldsymbol{z}^{i}\left(t_{k}\right) \mid \beta\right)=\exp \left(\left(z^{i}-w\right) / \sigma^{2}\right),
$$

where $\sigma^{2}$ is the variance of the measurement noise (chosen based on sensor characteristics).

To validate the Bayesian methodology and evaluate the performance of the generalized flow model, a series of ground-based experiments were conducted. A common 32 inch-diameter household fan, recast as a source of vertical flow disturbance, generated the flow. A test stand suspended the airspeed probes over the vertical source while data from an OptiTrack motion-capture system provided position information. To test the methodology in a more complex flow field than was modeled, experiments were conducted within the zone of flow establishment where velocity profiles have twin velocity peaks near the center line. ${ }^{27}$ Even though this flow feature was unmodeled, a series of localization tests were successfully carried out using a single probe, followed by a set of tests with a quadrotor mockup. The quadrotor mock-up stand is shown in Fig. (4). Velocity measurements were taken at a number of probe locations, simulating a series of trajectories within the flow field. The spinning rotors simulated the self-noise generated in flight.

The idealized flow model and Bayesian framework provided accurate fan location estimates. The algorithm typically estimated the center of the fan to within $2 \mathrm{~cm}$, or under $3 \%$ of the fan diameter. Results from a sample test run are presented in Fig. (4) showing estimates within $2 \%$ of fan diameter in four time steps. The colormap shows the posterior distribution of the location of the fan, with red indicating high probability and blue indicating low probability. The green and red asterisks denote probe location and the red circle indicates the actual location of the fan, unknown to the estimator. 


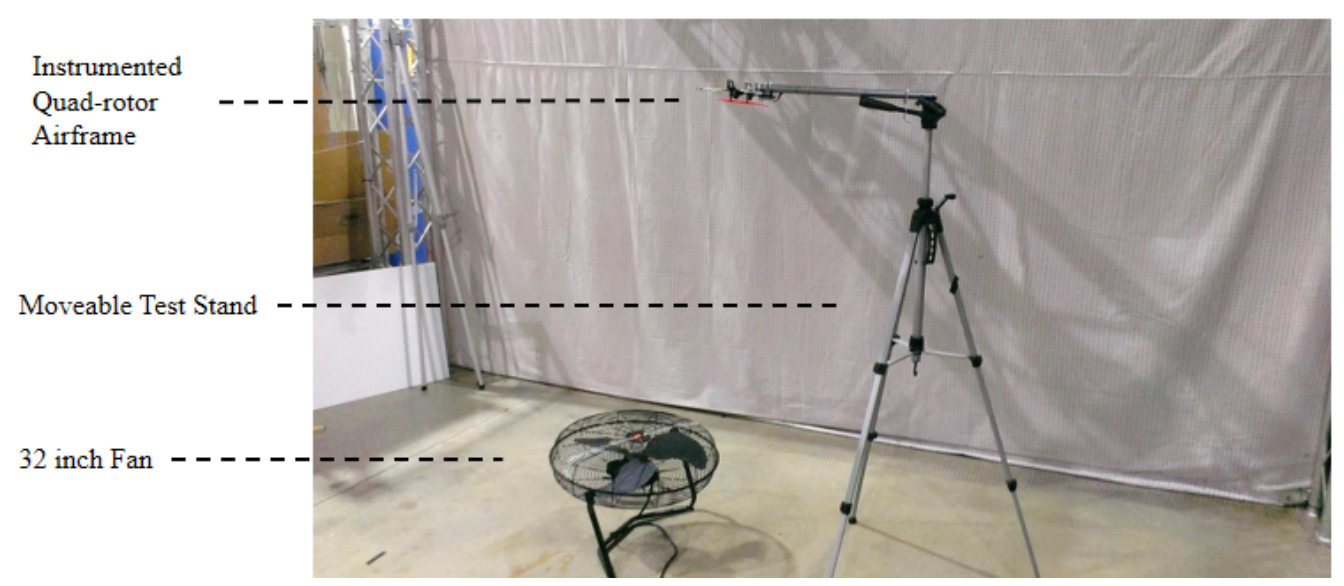

(a) Test Setup

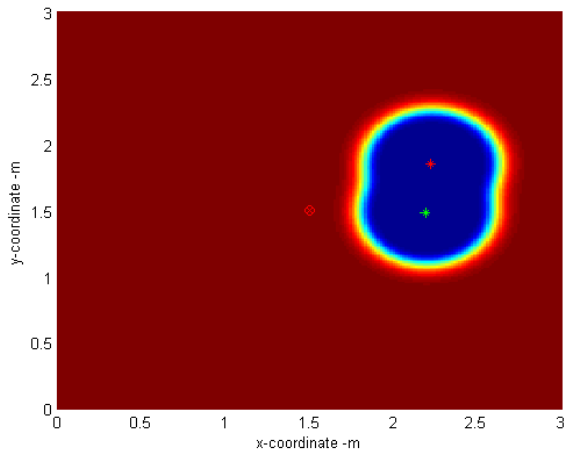

(b) Position 1

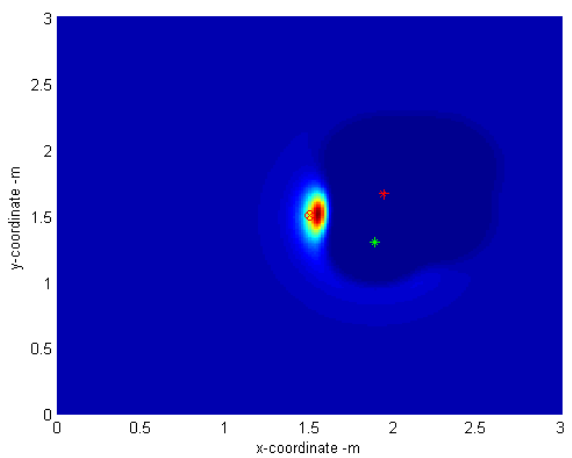

(d) Position 3

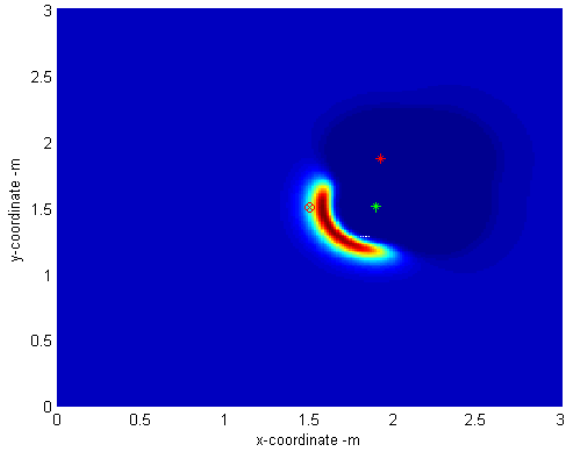

(c) Position 2

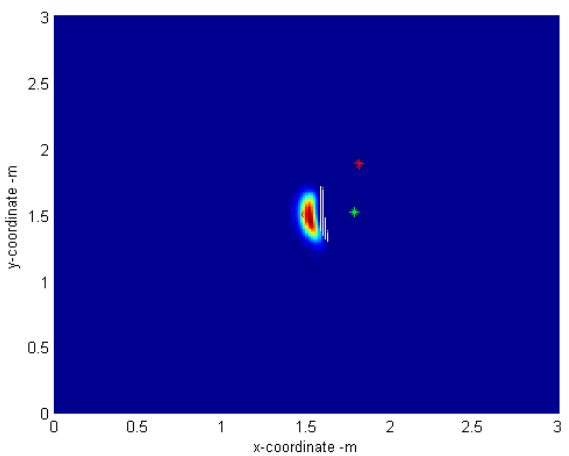

(e) Position 4

Figure 4. Quadcopter mock-up and sample localization results

\section{Proximity Flight Experimental Results}

This section showcases the utility of a flow sensing and control system for proximity flight. The scenario involves multiple quadrotors operating at different altitudes. The downwash of a quadrotor causes extreme, undesired changes in attitude and altitude of a quadrotor flying below. ${ }^{21}$ The flight-path planner and flow measurement system were implemented on the lower quadrotor. The planner generates an estimate of the position of the higher quadrotor to determine the direction the lower quadrotor must travel to reach a desired waypoint while avoiding the downwash. The flow measurement system and path planner were validated in simulation and experiment. 
Consider a vehicle with the dynamics

$$
\begin{aligned}
\dot{x} & =u_{x} \\
\dot{y} & =u_{y},
\end{aligned}
$$

where $x$ and $y$ are the coordinates the vehicle and $u_{x}$ and $u_{y}$ are control inputs. The goal is to design $u_{x}$ and $u_{y}$ to drive the vehicle to a waypoint and avoid the vertical jet produced by the higher quadrotor. The cost function $J\left(x, y, t_{k}\right)$ incorporates the desired goal and the presence of a vertical flow field, i.e.,

$$
J\left(x, y, t_{k}\right)=p\left(\overline{\boldsymbol{z}}\left(t_{k}\right) \mid \beta\right)+k_{J} \sqrt{\left(x-x_{d}\right)^{2}+\left(y-y_{d}\right)^{2}},
$$

where $\left(x_{d}, y_{d}\right)$ is the location of the goal and $k_{J}$ is a weighting variable. Intuitively, the cost is high when the vehicle is far away from the waypoint and/or near the downwash of the higher quadrotor. The goal is to find a path $C$ through the domain such that the integral of $J$ along the path is locally minimized. Formally, the problem is stated as

$$
\begin{gathered}
\underset{u_{x}, u_{y}}{\operatorname{minimize}} \quad \int_{C} J\left(x, y, t_{k}\right) d s \\
\text { subject to } \\
\dot{x}=u_{x} \\
\dot{y}=u_{y},
\end{gathered}
$$

where $d s$ is an increment along the path.

To decrease the computational complexity, a receding horizon version of (13) looks only one time step ahead. In this case the cost function reduces to

$$
J_{R H}=J\left(x, y, t_{k}\right)+J\left(x+\Delta x, y+\Delta y, t_{k}\right)
$$

Since the first term on the right-hand side is fixed by the current vehicle location, the cost function is minimized by moving in the direction of greatest decrease. Thus, in the zero limit of $\Delta x$ and $\Delta y,(14)$ is minimized using the control

$$
\begin{aligned}
& u_{x}=-K \frac{\partial J}{\partial x} \\
& u_{y}=-K \frac{\partial J}{\partial y},
\end{aligned}
$$

where $K$ is a control gain. This choice of control moves the vehicle in the direction of greatest decrease in cost.

The algorithm was first tested in simulation. The hovering quadrotor was commanded to hover at an altitude of $2.5 \mathrm{~m}$, and the instrumented quadrotor was given a waypoint at $(2.0,0.0) \mathrm{m}$. Fig. 5 shows the results of the algorithm. Each subfigure shows a snapshot of the simulation at a different timestep, starting from the initial time until the vehicle reaches the goal. The colormap at the bottom shows the posterior distribution for the position of the hovering quad, with red and blue denoting high and low probability respectively. The black dot indicates the position of the hovering quadrotor, the green dot is the position of the instrumented quadrotor, and the red $\mathrm{X}$ shows the destination of the instrumented vehicle. The trajectory of the quadrotor is shown as a white line on top of the posterior distribution. The estimate of the position of the hovering quadrotor is shown as a magenta dot. The results show that the instrumented quadrotor travels towards the waypoint, then diverts once it detects the higher quadrotor using simulated flow measurement data. Note that the estimate does not achieve zero steady-state error due to noise in the sensor measurements.

To validate the flow measurment system and proximity flight path planner, experiments were performed at the Naval Research Laboratory in the Laboratory for Autonomous Systems Research (LASR). The experiments were performed in a motion-capture testbed in the Prototyping Highbay at LASR, which is 150 by $75 \mathrm{ft}$ and equipped with 115 Vicon motion-capture cameras. Flight tests were conducted using two Ascending Technologies Pelican quadrotors. The Pelican has two onboard computers, one for flight stabilization and a Linux computer for sensor integration and control calculations. The Linux computer runs the Robot Operating System (ROS), which is a message-passing architecture for autonomous robots. 


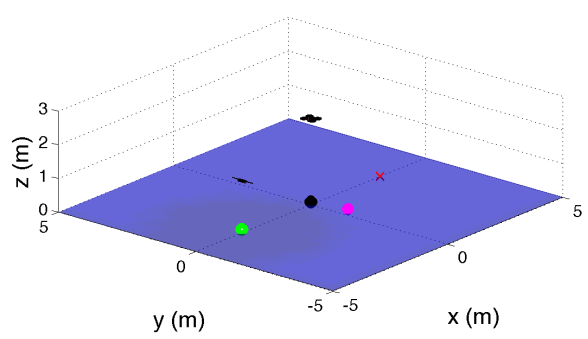

(a) $\mathrm{t}=0 \mathrm{~s}$

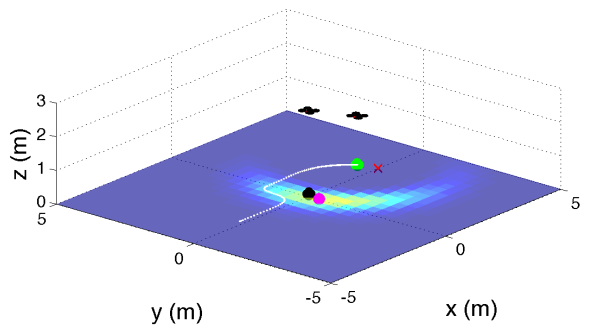

(c) $\mathrm{t}=10 \mathrm{~s}$

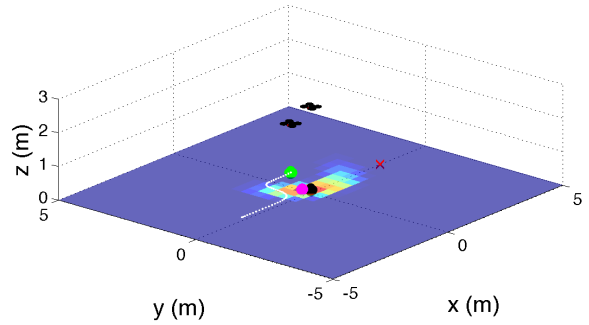

(b) $\mathrm{t}=5 \mathrm{~s}$

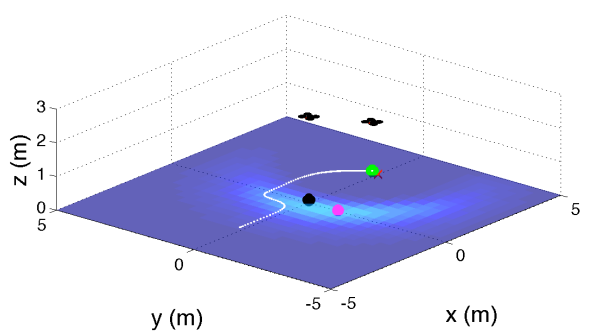

(d) $\mathrm{t}=15 \mathrm{~s}$

Figure 5. Trajectory of an instrumented quadrotor in proximity flight. The colormap indicates the posterior distribution of the probability of the hovering quadrotor location.

In the experiment, the high-altitude quadrotor was commanded to hover at the origin at an altitude of $2.5 \mathrm{~m}$. The instrumented quadrotor was commanded to go to the waypoint $(2.5,0.0) \mathrm{m}$ while an altitude of $1.5 \mathrm{~m}$ from five separate initial conditions. Fig. 6 shows the results of the flight test for all of the runs. Fig. 6(a) shows the trajectory of two quadrotors. The black $\mathrm{X}$ indicates the position of the hovering quadrotor and the green $\mathrm{X}$ shows the desired waypoint. The dashed black circle shows the approximate area where the downwash of the hovering quadrotor is significant. The dashed red trajectory shows the path of the vehicle with the flow measurement system and control disabled to show the nominal trajectory the vehicle takes. (Note that for safety reasons the nominal trajectory was implemented without the presence of the hovering quadrotor.) The other trajectories show the quadrotor with the flow measurement system and control enabled. Fig. 6(b) shows the measurements (colored dots) taken by the flow measurement system for the red colored run as well as the estimation error (solid lines) of the hovering quadrotor's position for all five runs that included the hovering vehicle (the estimates are color coded to match the trajectories in Fig. 6(a)). Fig. 6(c) shows the $x$ and $y$ position estimates of the hovering quadrotor for the red run.

The experiments illustrates the necessity of the flow measurement system and the flight-path planner. A vehicle without the combined system travels in a straight trajectory towards the waypoint, as indicated by the dashed red line in Fig. 6(a). This trajectory would place the vehicle directly in the downwash of the higher quadrotor. The vehicle with the flow measurement and flight-path planner detects the downwash of the higher quadrotor at approximately $t=3.0$ seconds for the red, blue, and green trajectories, as seen in Fig. 6(b). As soon as the sensor measures a positive airspeed, the error in the estimate of the position of the higher quadrotor drops to close to the desired position. Note that the error in the estimate is likely due to a combination of sensor noise and uncertainty in the choice of parameters in the likelihood function. Once the estimate of the higher quadrotors position converges, the instrumented vehicle maneuvers to avoid the downwash, as shown by the red, blue, and green trajectory in Fig. 6(a). The position estimates for the red run seen in Fig. 6(c) show how the estimate converges to near the correct value when the instrumented 


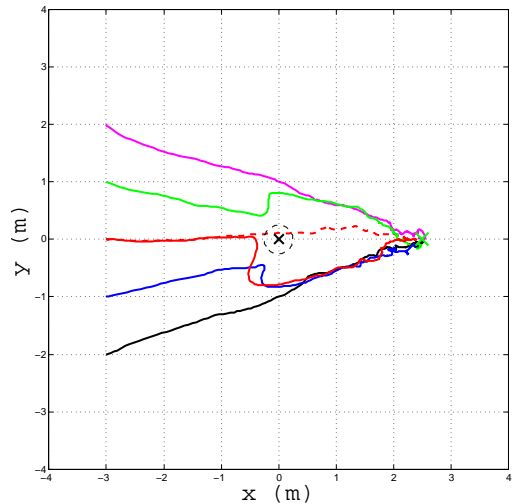

(a)

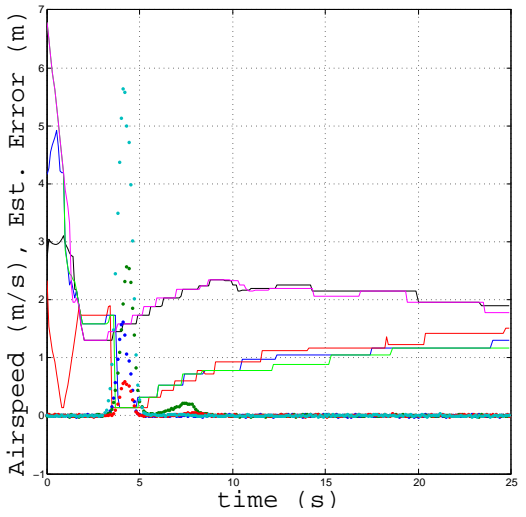

(b)

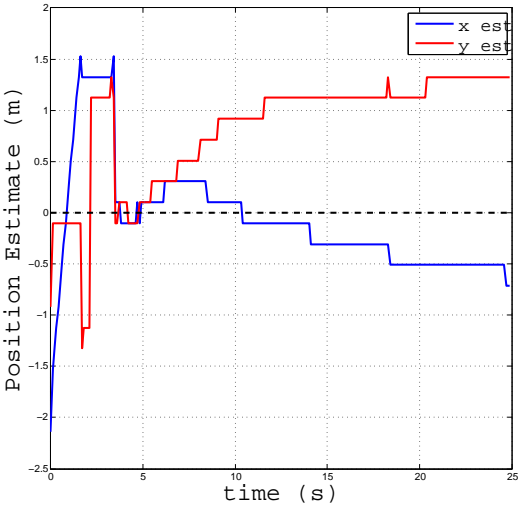

(c)

Figure 6. (a) Trajectory of the quadrotor from five separate initial conditions with the hovering vehicle located at the origin and one initial condition without the hovering vehicle. (b) measurements from the airspeed probes from red trial and hovering quadrotor estimation error for all five runs; (c) position estimate of the hovering quadrotor for the red run (middle trajectory)

vehicle passes close to the hovering quadrotor. Note that the estimate drifts away once the downwash is no longer detected, due to process noise that is added to the recursive Bayesian filter to avoid probability collapse. The magenta and black trajectories do not travel close enough to the hovering quadrotor to even detect the downwash. This is apparent in both Figures 6(a) and 6(b) as the vehicle does not maneuver to avoid the downwash and the estimate never converges.

\section{Conclusion}

An onboard flow sensing concept for quadrotor platforms is introduced along with an instrumentation package based on a set of custom wind-velocity probes. Data from ground-based tests and autonomous flight trials show that the system is capable of providing accurate wind-vector measurements in flight. A flight path planner is designed to use these measurements to generate an estimate of the surrounding flow field in the presence of a second rotorcraft; safe trajectories avoid hazardous downwash conditions. The flow measurement system and flight-path planner were tested in simulation and in a motion-capture testbed. Experiments showed that the vehicle was able to successfully avoid the downwash of another quadrotor and reach a desired goal. Ongoing work aims to develop an autopilot that incorporates flow-speed measurements into an attitude stabilization controller.

\section{Acknowledgments}

This work was performed at the University of Maryland and the Naval Research Laboratory and was funded by the US Department of Defense, Office of Naval Research under grant number N0001413WX21045, Mobile Autonomous Teams for Navy Information Surveillance and Search (MANTISS); U. S. Army under Grant No. W911W6112072; and the Air Force Office of Scientific Research under Grant No. FA95501310162, Dynamic Data Driven Applications Systems (DDDAS). The views, positions and conclusions expressed herein reflect only the authors opinions and expressly do not reflect those of the US Department of Defense, Office of Naval Research, or the Naval Research Laboratory.

\section{References}

${ }^{1}$ M. Belkheri, A. Rabhi, A. Hajjaji, and C. Pergard. Different linearization control techniques for a quadrotor system. In 2nd Int. Conf. on Communications, Computing and Control Applications,, pages 1-6, December 2010.

${ }^{2}$ D. Alexander and S. Vogel. Nature's Flyers: Birds, Insects, and the Biomechanics of Flight. Johns Hopkins University Press, October 2004.

${ }^{3} \mathrm{M}$. Gewecke and Martin Woike. Breast feathers as an air-current sense organ for the control of flight behaviour in a songbird (carduelis spinus). In Zeitschrift fr Tierpsychologie ,, volume 47, pages 293-298, 1978. 
${ }^{4}$ R. Brown and M. Fedde. Airflow sensors in the avian wing. In Journal of Experimental Biology ,, volume 179, pages 13-30, 1993.

${ }^{5}$ S. Herwitz, K. Allmendinger, R. Slye, S. Dunagan, B. Lobitz, L. Johnson, and J. Brass. Nighttime UAV vineyard mission: Challenges of see-and-avoid in the NAS. In Proc. AIAA 3rd Unmanned Unlimited Conference, Workshop and Exhibit, pages 1-6, September 2004.

${ }^{6}$ R. Beard, D. Kingston, M. Quigley, D. Snyder, R. Christiansen, W. Johnson, T. McLain, and M. Goodrich. Autonomous vehicle technologies for small fixed wing UAVs. In AIAA Journal of Aerospace Computing, Information, and Communication, volume 2, page 92, January 2005.

${ }^{7}$ R. Hirokawa, D. Kubo, S. Suzuki, J. Meguro, and T. Suzuki. Small UAV for immediate hazard map generation. In $A I A A$ Infotech@Aerospace Conf, May 2007.

${ }^{8}$ F. Hsiao, Y. Ding, C. Chuang, C. Lin, and Y. Huang. The design of a small UAV system as a testbed of formation flight. In AIAA Infotech@Aerospace Conf, March 2011.

${ }^{9}$ R. Eubank, E. Atkins, and D. Macy. Autonomous guidance and control of the flying fish ocean surveillance platform. In AIAA Infotech@Aerospace Conf, April 2009.

${ }^{10} \mathrm{~N}$. Rasmussen, B. Morse, and C. Taylor. Fixed-wing, mini-UAV system for aerial search operations. In AIAA Guidance Navigation and Control Conference and Exhibit, August 2007.

${ }^{11}$ P. Xie, A. Flores-Abad, G. Martinez, and O. Ma. Development of a small UAV with autopilot capability. In Proc. AIAA Atmospheric Flight Mechanics Conference, August 2011.

${ }^{12}$ M. Patel, Z. Sowle, T. Corke, and C. He. Autonomous sensing and control of wing stall using a smart plasma slat. In Proc. 44th AIAA Aerospace Sciences Meeting, January 2006.

${ }^{13}$ P.Bowles and T. Corke. Stall detection on a leading-edge plasma actuated pitching airfoil utiliizing onboard measurement. In Proc. 47th Aerospace Sciences Meeting, January 2009.

${ }^{14}$ Y. Xu, F. Jiang, S. Newbern, A. Huand, C. Ho, and Y. Tai. Flexible shear-stress sensor skin and its application to unmanned aerial vehicles. In Sensors and Actuators A: Physical, volume 105, pages 321-329, 2003.

${ }^{15}$ C. Gorsjean, G. Lee, W. Hong, Y. Tai, and C. Ho. Micro balloon actuators for aerodynamic control. In Micro Electro Mechanical Systems, The Eleventh Annual International Workshop on, pages 166-171. IEEE, January 1998.

${ }^{16}$ Air Force Office of Scientific Research (AFOSR). Multidisciplinary university research initiative (muri).

${ }^{17}$ W. Barnwell. Flight Control Using Distributed Actuation and Sensing. Master's thesis, North Carolina State University, USA, 2003.

${ }^{18}$ S. Lion. Control Authorities of a distributed Actuation and Sensing Array on a Blended-Wing-Body Uninhabited Aerial Vehicle. Master's thesis, North Carolina State University, USA, 2007.

${ }^{19}$ C. Hall. C. Cox, A. Gopalarathnam. Flight test of stable automated cruise flap for an adaptive wing aircraft. In Journal of Aircraft, volume 47, pages 1178-1188, January 2009.

${ }^{20}$ D. Yeo, E. Atkins, L. Bernal, and W. Shyy. Aerodynamic sensing for a fixed wing uas operating at high angles of attack. In Proc. AIAA Atmospheric Flight Mechanics Conference, August 2012.

${ }^{21}$ N. Sydney, B. Smyth, and D. A. Paley. Dynamic control of autonomous quadrotor flight in an estimated wind field. In Decision and Control (CDC), 2013 IEEE 52nd Annual Conference on, pages 3609-3616, Dec 2013.

${ }^{22}$ K. Alexis, G. Nikolakopoulos, and A. Tzes. Experimental model predictive attitude tracking control of a quadrotor helicopter subject to wind-gusts. In 18th Mediterranean Conf. on Control Automation (MED), 2010, pages 1461 -1466, June 2010.

${ }^{23}$ K. Alexis, G. Nikolakopoulos, and A. Tzes. Constrained-control of a quadrotor helicopter for trajectory tracking under wind-gust disturbances. In MELECON 2010, pages 1411-1416, April 2010.

${ }^{24}$ C. Powers, D. Mellinger, A. Kushleyev, B. Kothmann, and V. Kumar. Influence of aerodynamics and proximity effects in quadrotor flight. In Proc. of the Int. Symp. on Experimental Robotics, June 2012.

${ }^{25}$ F. White. Viscous Fluid Flow. McGraw-Hill Mechanical Engineering, 3 edition, January 2005.

${ }^{26}$ R. Jensen M. Albertson, Y. Dai and H. Rouse. Diffusion of submerged jets. In Transactions of the American Society of Civil Engineers, volume 115, pages 639-697. American Society of Civil Engineers, 1950.

${ }^{27}$ W. Khan, R. Caverly, and M. Nahon. Propeller slipstream model for small unmanned aerial vehicles. In AIAA Modelling and Simulation Conference. AIAA, August 2013. 